\title{
Anstellungsbedingungen der Kaderärztinnen und Kaderärzte an öffentlichen und öffentlich subventionierten Spitälern
}

\author{
Prof. Dr. Alex Angehrn ${ }^{a}$, Dr. T. Eichenberger ${ }^{b},{\text { Franz } W y s s^{c}}^{c}$
}

Die Private Hochschule Wirtschaft PHW (ein Teil der Berner Fachhochschule) hat im Herbst 2003 im Auftrag der Schweizerischen Sanitätsdirektorenkonferenz SDK (heute GDK) sowie des Vereins der Leitenden Spitalärzte der Schweiz (VLSS) eine umfangreiche Befragung bei den Kaderärztinnen und Kaderärzten sowie bei einigen öffentlichen Spitälern der Schweiz durchgeführt und ausgewertet.

\footnotetext{
a Ehem. Direktor der Privaten Hochschule Wirtschaft PHW, Bern

b Geschäftsleiter Verein der Leitenden Spitalärzte der Schweiz VLSS, Bern

c Zentralsekretär Schweizerische Konferenz der kantonalen Gesundheitsdirektorinnen und -direktoren GDK, Bern
}

\section{Zielsetzung}

Mit den aus dieser Befragung resultierenden Informationen wollten die Auftraggeber eine bessere Grundlage für die laufenden Diskussionen um die zukünftige Gestaltung der Anstellungsbedingungen der Kaderärztinnen und Kaderärzte in den öffentlichen und subventionierten Spitälern bereitstellen. Bisher lagen darüber keine gesicherten wissenschaftlichen Erkenntnisse vor; es fehlte insbesondere an der Vergleichbarkeit zwischen den einzelnen Kantonen. Die Datenlage hat sich mit der Durchführung der erwähnten Studie, insbesondere dank der kooperativen Mitarbeit der öffentlichen Spitäler und der Kaderärztinnen und Kaderärzte, wesentlich verbessert. Die Studie wird, weil teilweise sensible Daten beinhaltend, nicht als Ganzes veröffentlicht. Wir ziehen es vielmehr vor, der geneigten Leserschaft, insbesondere den Umfrageteilnehmerinnen und Umfrageteilnehmern, die wichtigsten Ergebnisse hier vorzustellen:

Wir sind darüber hinaus auch gerne bereit, weiterführende gezielte Anfragen von interessierten Umfrageteilnehmerinnen und -teilnehmern zu beantworten, sofern und soweit es die Datenlage zulässt.

\section{Rücklauf der Fragebogen, Einkommen, Arbeitszeit}

\section{Rücklauf}

Von 2838 Ärztefragebogen konnten 638 ausgewertet werden. Dies entspricht einem Rücklauf von $22 \%$, der bei Ärztebefragungen mit sensiblen Fragestellungen ungefähr den Erwartungen entspricht. Die Verteilung der Antworten (Abb. 1) zeigt, dass - entsprechend der zurzeit noch vorhandenen Untervertretung in dieser Berufsgruppe - wesentlich weniger Frauen $(\mathrm{N}=183)$ als Männer $(\mathrm{N}=579)$ geantwortet haben. Der Stichprobenumfang war für einige Rubriken («CoChefärzte» $[\mathrm{N}=41]$, «Andere Ärzte» $[\mathrm{N}=21]$, «Unter 35 Jahren» $[\mathrm{N}=2]$, «Psychiatrische Institutionen» $[\mathrm{N}=34]$ und «Andere Spitalarten» $[N=57]$ ) sehr gering, so dass für diese Funktionsbereiche aus statistischen Gründen keine separaten Auswertungen vorgenommen werden konnten.

Um die gesamte Untersuchung breiter abzustützen, wurden nicht nur die Ärzte befragt, sondern ausgewählte Spitäler in die Untersuchung miteinbezogen. Insgesamt wurden 22 repräsentative Spitäler angeschrieben. 15 Spitäler haben den Fragebogen, der gleichzeitig mit dem Ärztefragebogen verschickt wurde, mehr oder weniger vollständig ausgefüllt. Damit war es möglich, die Angaben der Kaderärztinnen und Kaderärzte zu plausibilisieren.

\section{Einkommen}

Das Minimum des Grundlohnes liegt gemäss den Angaben der Spitäler bei Fr. 128000.(Minimum Leitende Ärzte) und das Maximum bei Fr. 219 000.- (Maximum Chefärzte). Der Vergleich des Einkommens aus Spitalanstellung und aus privatärztlicher Tätigkeit aus der Ärztebefragung (Abb. 2) bestätigt diese Angaben, zeigt aber auch, dass zusätzlich Einkommensbestandteile in vergleichbarem Umfang aus privatärztlicher Tätigkeit dazukommen.

Der regionale Vergleich verdeutlicht, dass das Gesamteinkommen der Kaderärztinnen und -ärzte in der Innerschweiz sowie in der Nordostschweiz am höchsten, dagegen in den französischsprachigen Kantonen und im Tessin am tiefsten ist. Die Nord-/Nordwestschweiz mit den Kantonen Bern, Solothurn, Aargau sowie BaselStadt und Basel-Landschaft liegt dazwischen. Die Umfrage bestätigt im weiteren, dass Chefärztinnen eher mehr verdienen als Leitende Ärzte sowie dass operativ oder interventionell tätige Kaderärzte mehr verdienen als weder operativ 
Abbildung 1

Übersicht Versand/Rückläufe.

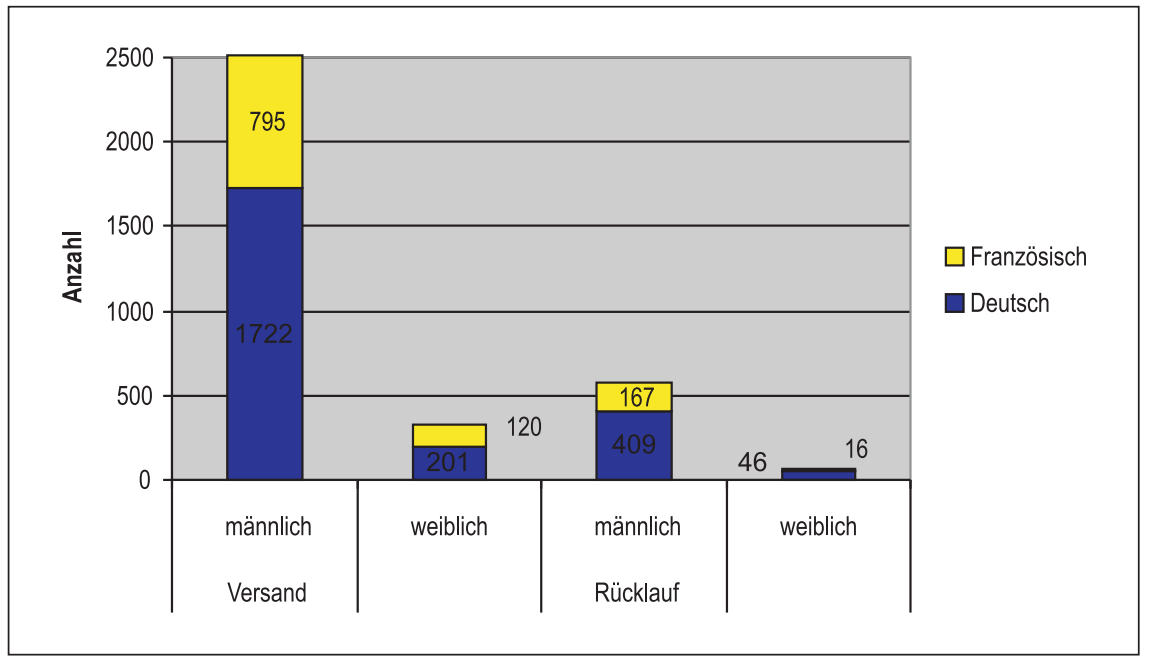

Abbildung 2

Vergleich Spitalanstellung und Honorare.

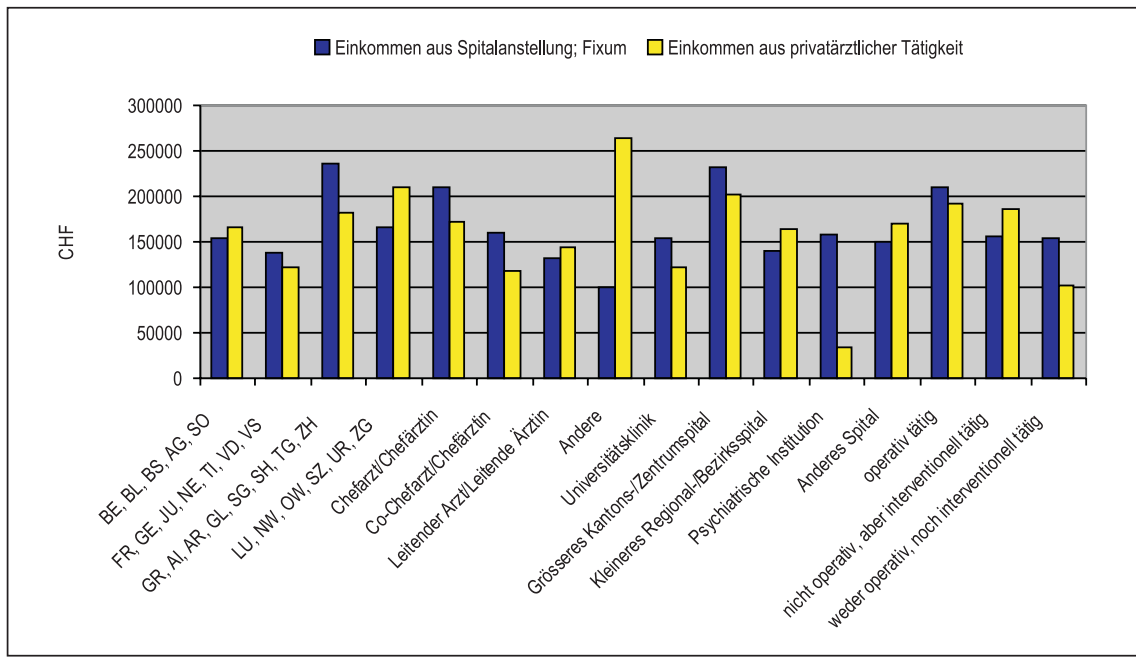

Abbildung 3

Summarische und geschätzte wöchentliche Arbeitszeit im Vergleich.

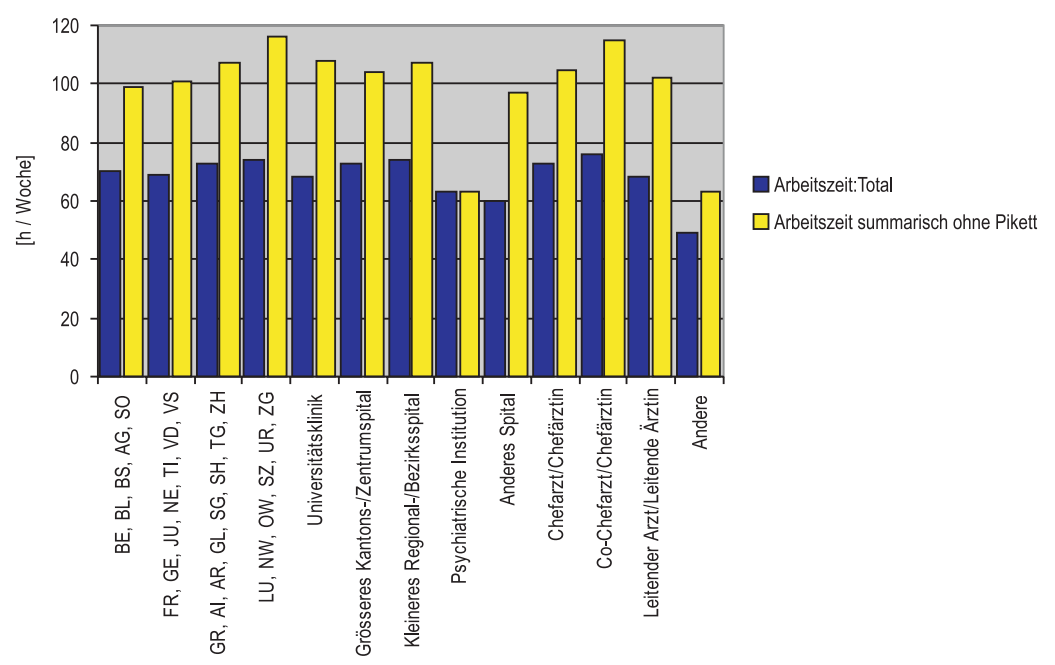

noch interventionell tätige Kaderärztinnen. Am wenigsten verdienen Kaderärzte an Psychiatrischen Institutionen. Dort ist die privatärztliche Tätigkeit gemäss den Umfrageergebnissen stark eingeschränkt. Vergleichbar sind dagegen die Einkommen an Universitätsspitälern und kleineren Regional-/Bezirksspitälern, während die höchsten Einkommen offenbar an grösseren Kantonsspitälern erwirtschaftet werden.

\section{Arbeitszeit}

Die Arbeitszeit spielt im Zusammenhang mit den Anstellungsbedingungen eine wichtige Rolle. Im Rahmen der vorliegenden Untersuchung wurden die Kaderärztinnen und Kaderärzte aufgefordert, ihre durchschnittliche wöchentliche Arbeitszeit während der letzten sechs Monate zu schätzen. Die wöchentliche Arbeitszeit beläuft sich demzufolge auf durchschnittlich rund 70 Stunden (Abb. 3). Andererseits hat die PHW aber auch die selbstdeklarierten Einzeltätigkeiten erfragt. Hier zeigt sich eine auffallende und kaum erklärbare Diskrepanz bei den Angaben, indem die Summe der selbstdeklarierten Einzeltätigkeiten entgegen der geschätzten Gesamtsumme rund 100 Stunden ergibt. Überraschend bei diesen Resultaten ist zudem der geringe Anteil des angegebenen zeitlichen Aufwandes für Führung und Management von weniger als $10 \mathrm{~h}$ pro Woche.

\section{Honorareinnahmen aus privatärztlicher Tätigkeit, Pools, Managementausbildung}

Im folgenden werden wir aufzeigen, nach welchen Grundsätzen die Honorareinnahmen aus privatärztlicher Tätigkeit zugunsten des Spitals verwendet werden, wie stark hierbei sogenannte Pools verbreitet sind, wie die Arbeitszeit des Arztes mit seiner Funktion zusammenhängt und wie es um die Ausbildung der Ärzteschaft in Führung und Management bestellt ist.

\section{Honorareinnahmen aus privatärztlicher Tätigkeit zugunsten der Spitäler}

Die Honorareinnahmen aus der Behandlung von Privatpatienten werden zwischen Spital und Arzt aufgeteilt. Der Arzt erhält eine Entschädigung für seine persönlich geleistete Arbeit, das Spital für die Bereitstellung der Infrastruktur und des Personals sowie für die administrative $\mathrm{Ab}$ wicklung der Zahlungen. Generell ist der Anteil des Spitals um so grösser, je höher das Gesamteinkommen des Arztes bzw. die Honorareinkünfte aus der privatärztlichen Tätigkeit sind. Abbildung 4 verdeutlicht zudem die regionalen 
Abbildung 4

Honorareinnahmen aus der privatärztlichen Tätigkeit zugunsten des Spitals.

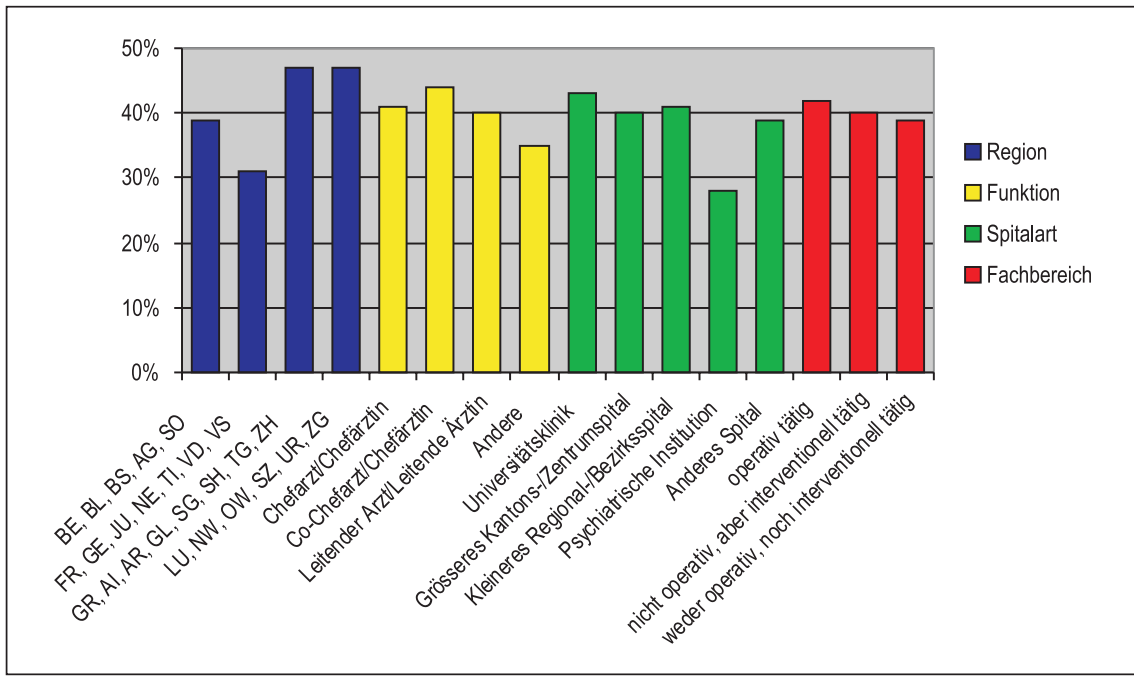

Abbildung 5

Existenz von Pools.

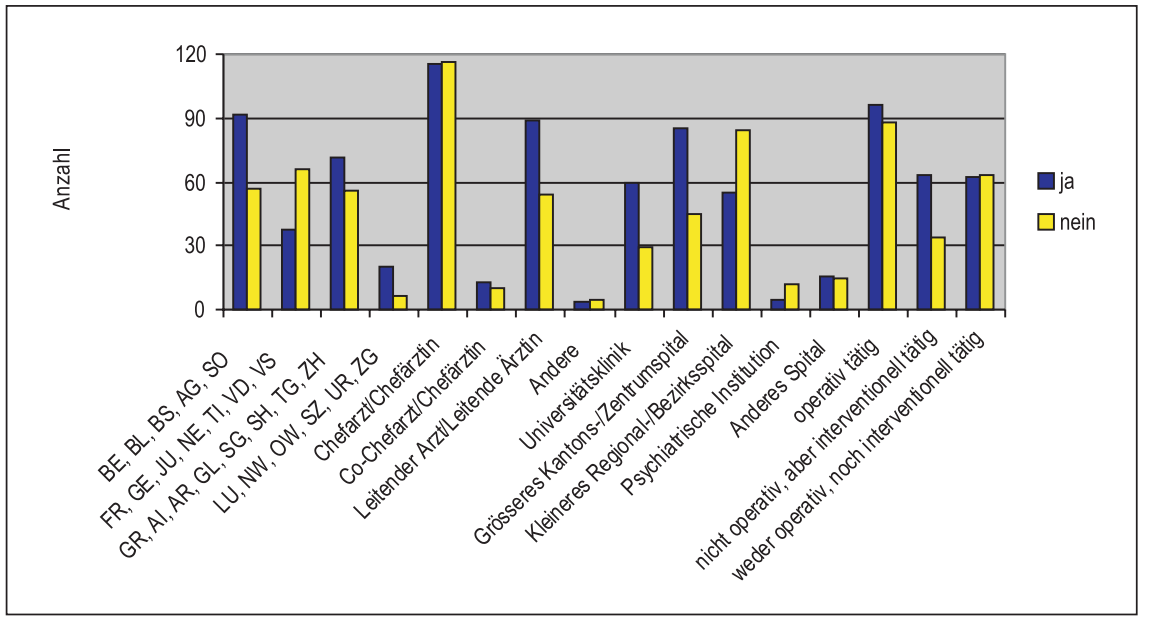

Abbildung 6

Arbeitszeit nach Funktion.

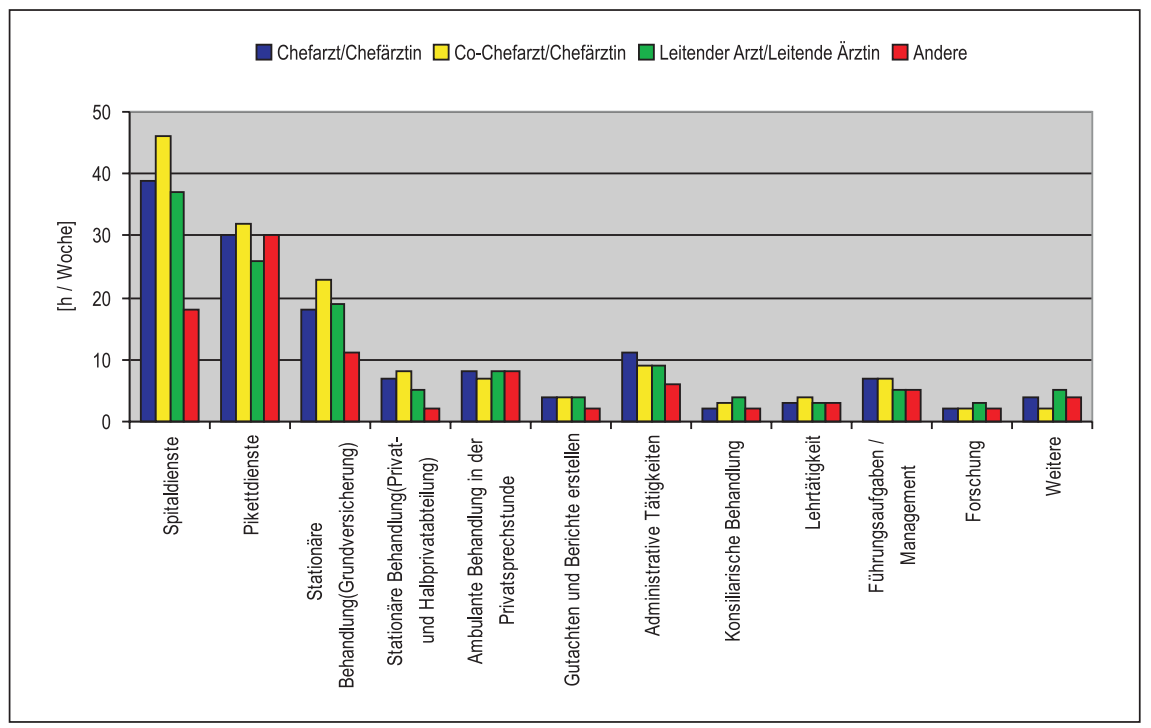

Unterschiede des Umfangs der Abgaben: Während in der lateinischen Schweiz der Anteil der abzugebenden Honorareinnahmen bei durchschnittlich $31 \%$ liegt, betragen die Werte für die Zentral- und Ostschweiz im Durchschnitt je $47 \%$. Der Wert für die Nord-/Nordwestschweiz liegt mit durchschnittlich 39\% in der Mitte.

Die Ermittlung der Spitalanteile basiert in der Regel auf vier verschiedenen Systemen: effektive Unkosten, Pauschalen, lineare und progressive Anteile. Obwohl überall in der Schweiz alle Systeme angewendet werden, zeigt die Studie auch hier regionale Unterschiede auf. Progressive Anteile überwiegen in der lateinischen Schweiz und der Zentralschweiz, bei den Chefärztinnen und -ärzten, bei den Universitätskliniken, den grösseren Kantons- und kleineren Regionalspitälern sowie bei den operativ und nicht operativ, aber interventionell tätigen Kaderärztinnen und Kaderärzten. Pauschale Anteile dominieren hingegen in der Nord-/Nordwestschweiz (BE, BL, BS, AG, SO), bei den Co-Chefärztinnen und -ärzten, bei den psychiatrischen Institutionen und bei den weder operativ noch interventionell tätigen Kaderärztinnen und -ärzten.

\section{Existenz von Pools}

Neben den Anteilen, die dem Spital infolge Nutzung von Infrastruktur (Inanspruchnahme von Räumen, Büromaterial, Porti, Telefon und Inkassospesen) und Beanspruchung von Personal zufliessen, werden Anteile zur Speisung von Pools und Fonds verwendet. Gemäss der Umfrage finden solche Pools in der Deutschschweiz offenbar mehr Anklang als in der Romandie; zudem bestehen sie häufiger an Universitätskliniken und grösseren Spitälern als an Regionalspitälern (Abb. 5).

Die Poolgelder werden am häufigsten für Aus- und Weiterbildungen sowie für die Beteiligung von Assistenz- und Oberärztinnen und -ärzten an den Honoraren aus privatärztlicher Tätigkeit verwendet. Einzig an Universitätskliniken kommen die Gelder in erster Linie der Lehre und Forschung zu.

\section{Arbeitszeit nach Funktion}

Ein wesentlicher Bestandteil der Anstellungsbedingungen betrifft die Arbeitszeit sowohl in ihrer gesamten Summe als auch bei den Hauptaufgaben. Wie bereits im letzten Artikel vermerkt, ergeben die nach Funktionen angegebenen, zusammengerechneten Stundenzahlen ein durchschnittliches Total von 100 Wochenstunden, was auf eine Überschätzung hindeuten könnte, nachdem die durchschnittlich geschätzte wöchentliche Gesamtarbeitszeit lediglich 70 Stunden be- 
Abbildung 7

Ausbildung in Führung und Management.

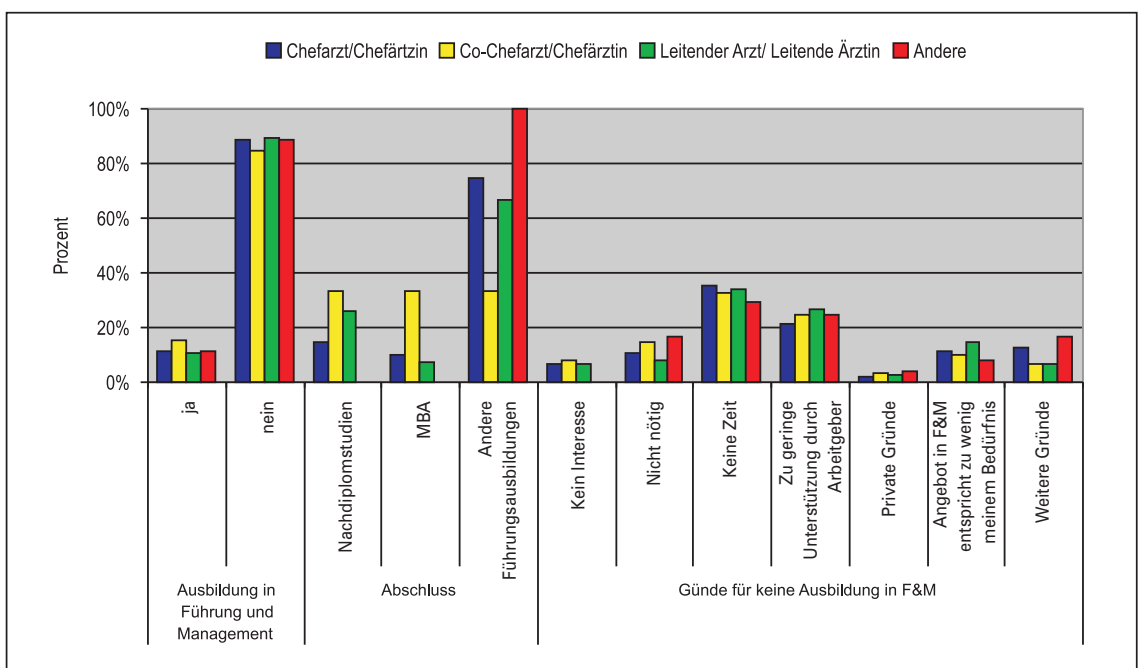

trägt. Die betreffenden Angaben in Abbildung 6 bieten deshalb lediglich Hinweise auf die ungefähre relative Bedeutung der einzelnen Tätigkeiten. Auffallend an den Resultaten ist, dass die funktionsbedingten Unterschiede nicht besonders gross sind: Die zeitliche Belastung für die Tätigkeit ist in allen Bereichen - unabhängig von der jeweiligen Funktion der Ärztin bzw. des Arztes - ungefähr gleich hoch. Auch Chefärztinnen und -ärzte leisten im übrigen Pikettdienst. Auf den geringen Anteil an Management- und Führungsaufgaben an der Gesamtarbeitszeit kommen wir sogleich zu sprechen.

\section{Ausbildung in Führung und Management}

Wie im ersten Teil dieses Artikels aufgezeigt, liegt der durchschnittlich angegebene zeitliche Aufwand für Führungs- und Managementtätigkeiten überraschenderweise unter 10 Stunden pro Woche. Dies könnte auch damit zusammenhängen, dass nur eine sehr geringe Anzahl von Ärztinnen und Ärzten überhaupt über eine entsprechende Ausbildung verfügt: Wie aus Abbildung 7 hervorgeht, sind es lediglich 12\% der befragten Kaderärztinnen und -ärzte. Die Aus- bildung bestand bei einem Drittel der Ausgebildeten in einem Nachdiplomstudium oder MBA, zwei Drittel absolvierten andere Führungsausbildungen wie militärische Kaderausbildungen, Managementseminare an Hochschulen und Universitäten sowie spitalinterne Führungskurse. Befragt nach den Gründen, weshalb aktive Kaderärztinnen und -ärzte nicht über einen Abschluss in Führung und Management verfügten, gaben $33 \%$ an, keine Zeit dafür zu haben; 24\% nannten eine $z u$ geringe Unterstützung durch den Arbeitgeber; 13\% fanden, das Angebot an Managementausbildungen entspreche nicht ihren Bedürfnissen; schliesslich meinten $10 \%$, eine derartige Ausbildung sei in ihrer Funktion gar nicht nötig.

\section{Schlussfolgerungen}

Die Anstellungsbedingungen der Kaderärztinnen und Kaderärzte zeigen, dass die Höhe des Einkommens nicht von der Managementaufgabe, sondern vom Fachbereich, der Spitalart und der Region abhängt. Ausserdem wird die hohe zeitliche Arbeitsbelastung empirisch erhärtet, wobei die ärztlichen Tätigkeiten weitaus überwiegen.

Weiter kann festgehalten werden, dass die Anstellungsbedingungen der Kaderärztinnen und Kaderärzte in der Schweiz trotz einer grossen Vielfalt zumindest in ihren Eckwerten vergleichbar sind. Obwohl der Individualitätsgrad der bestehenden Verhältnisse hoch ist und für viele Situationen spezifische Lösungen bestehen, sind gewisse Gesamtaussagen möglich, so z.B. zum Einkommen oder zur Ausbildung in Führung und Management. Das gesammelte Datenmaterial stellt deshalb eine wertvolle Grundlage für die vergleichende Beurteilung der Anstellungsbedingungen der Kaderärztinnen und Kaderärzte in der Schweiz dar und kann dazu dienen, bestehende Defizite besser zu erkennen. Darüber hinaus könnte es dazu beitragen, näher aufzuzeigen, wo eine allfällige Vereinheitlichung zumindest sinnvoll oder sogar notwendig wäre. 